\title{
The analysis of the relationship between personality traits of extreme athletes and sports consumption motives
}

\author{
Merve Ceylan ${ }^{1}$ \\ Ersin Altıparmak ${ }^{2}$ \\ Fahri Akçakoyun ${ }^{3}$
}

\begin{abstract}
This research was conducted in order to analyse the relationship between personality traits and sports consumption motives. In line with this purpose, 227 extreme athletes (86 were sportsmen and 141 were sportswomen), which were competitors in sports branches of snowboarding, snow kiting, kitesurfing, windsurfing, kayak, mountain biking, Free-diving, paragliding, rock climbing and rafting, were selected through the method of judgement sampling. Questionnaires were conducted between 1 February- 31 December 2013 at extreme sports facilities in the provinces and districts of Muğla/Marmaris-Fethiye-Bodrum-Gökova, Antalya/Alanya-Kemer, İzmir/Alaçatı-Çeşme, Bursa/ Uludağ, Bolu/ Kartalkaya. In this study, "Extreme Sport Consumption Scale" (ESCS) that was developed by Şimşek (2010) and short version (with 85 articles) of "Five Factor Personality Inventory" (FFPI), which was developed by Somer, Korkmaz and Tatar (2004) were employed in order to collect data.

The results of the study produced significant findings concerning the relationship between personality traits and their sports consumption motives. The most significant of them all showed that sports consumption motives to a certain extent coincide with personality traits. The assessment of the relationship between ESCS and FFPI according to the gender factor revealed that for female participants, there were negative correlations between physical fitness and extraversion; values, socialisation, seeking for excitement-adventure and docility and letting off stress and self-control $(\mathrm{p}<.05)$. For male participants, positive correlations were observed between letting off stress, behavioural intentions and extraversion $(\mathrm{p}<.05)$.
\end{abstract}

Keywords: Extreme Sports; Extreme Athletes; Personality.

1Dr., Mugla Sttk1 Koçman University, Faculty of Sports Science, Physical Education and Sport, merveceylan@ymail.com

2Dr., Ege University, Physical Education and Sport Schools, Psychosocial Fields in Sports, ersin.altiparmak@ege.edu.tr ${ }^{3}$ Dr., Mugla Sttkı Koçman University, Faculty of Sports Science, Recreation, afahri@mu.edu.tr 
Ceylan, M., Altıparmak, E., \& Akçakoyun, F. (2016). The analysis of the relationship between personality traits of extreme athletes and sports consumption motives. International Journal of Human Sciences, 13(1), 1745-1754. doi:10.14687/ijhs.v13i1.3416

\section{Introduction}

As a part of struggle for survival in society, the obligation to work sometimes confines individuals in an office with desk-bound professions and leads to an inactive life; in some cases it causes exhaustion and negatively affects lives of individuals. Alongside with this way of live, which is not compatible with human nature, pressure and stress from business and social environments distress people psychologically. As a result individuals, who wear down at young ages, feel the need of regeneration; they attempt to let themselves be in nature and do extraordinary activities in order to move away from stress. Particularly in our country extreme sports have become popular in recent years and they have almost occupied the first rank in this regeneration process; this has led to increasing variety in extreme sports (Ceylan, 2010, 54).

As daily life is not dangerous or exciting, ordinary life stipulates dissatisfaction and stimulation of social needs and it is suggested that each individual can move away from this dissatisfaction by participating extreme activities (Palmer, 2002).

It is underlined that extreme sports have significant distinctive features from other types of sports that extreme sports make individuals to experience high risk perception, high concentration, excitement, adventure, tendency to freedom and different lifestyles. The basic feature that differentiates extreme sports from traditional sports is the death risk. Furthermore, extreme sports participants are said to be motivated through features such as high level of stimulation, adrenaline, aggression, excitement, struggle, uniqueness, psychological situation, physical/mental comfort and achievement (Şimşek, 2010, 98).

In extreme sports, taking risks voluntary is defined as a "behaviour including individualism"; it is participation to selective activities deliberately in which danger is perceived(Williams vet Gill, 2000). Individuals perceive humans, events and objects outside, as they perceive their own feelings and ideas. The personality of an individual determines the type of relationship that he or she establishes with his or her external environment (Cüceloğlu, 2007).

With regard to understanding of extreme sports consumer behaviour, perhaps one of the most important concepts is personality; it is also one of the hardest concepts to understand and to associate its current ties with sports consumption. In this sense personality, which constitutes a basis for and individual's behaviours, determines all behaviours of an individual; at the same time it might be considered to shape sports consumption behaviour. Incentive factors concerning participation to extreme sports and extreme sports consumption behaviour are the most important determinants in sports participation. Research on participation to extreme sports reveal several main motives such as letting off stress, mastership, aesthetics, self-realisation, self-esteem, value development, social relaxation, belonging/relationship, competition, aggression, taking risks, achievement, physical fitness and entertainment/pleasure (Şimşek, 2011, 134).

There are many studies in the sports literature dealing with extreme athletes, personality, motivation and risky sports. However no such a study analysing the impacts of personality traits on sports consumption motives has been found. Therefore, in order to fill this gap, the study analysed the relationship between personality and sports consumption motives.

\section{Material and Method}

The population of this research consists of extreme athletes, who benefit from extreme sports facilities in provinces and districts within the borders of Turkey. The sample of the study includes active participants or extreme athletes who currently train for a competition.

In this study, 227 extreme athletes (86 were sportsmen and 141 were sportswomen), which were competitors in sports branches of snowboarding, snow kiting, kitesurfing, windsurfing, kayak, mountain biking, Free-diving, paragliding, rock climbing and rafting, were selected through the method of judgement sampling. Questionnaires were conducted between 1 February- 31 December 2013 at extreme sports facilities in the provinces and districts of Muğla/Marmaris-Fethiye-BodrumGökova, Antalya/Alanya-Kemer, İzmir/Alaçatı-Çeşme, Bursa/ Uludăg, Bolu/ Kartalkaya. 
Ceylan, M., Altıparmak, E., \& Akçakoyun, F. (2016). The analysis of the relationship between personality traits of extreme athletes and sports consumption motives. International Journal of Human Sciences, 13(1), 1745-1754. doi:10.14687/ijhs.v13i1.3416

In this research, the researcher conducted questionnaires with participants face-to-face in extreme sports facilities. In an 11 month-period, data were collected through partially group activities and partially individual implementations consisting of athletes that voluntarily participate in survey. Collected data were recorded with SPSS software. Frequency, percentage, arithmetic mean and standard deviation values were calculated. "Independent samples t- test" was used to find out difference between genders and "Pearson correlation test" was employed to analyse the relationship between personality and sports consumption motives.

In this research, "Extreme Sport Consumption Scale" (ESCS) that was developed by Simşek (2010) and short version (with 85 articles) of "Five Factor Personality Inventory" (FFPI), which was developed by Somer, Korkmaz and Tatar (2004) were employed in order to collect data. Five Factor Personality Inventory provides total points concerning the properties of Extroversion, Docility/Agreeableness, Self-Control/Responsibility, Neuroticism and Openness to Experience.

Extraversion; frequently refers to traits such as being social, gregarious, assertive, challenging, active and talkative.

Docility; includes traits such as being kind, gentle, flexible, trustable, well mannered, prone to cooperation, forgiving, light-hearted and tolerant.

Self-control; refers to traits including being careful, attentive, thorough, responsible, well ordered, organised, achievement-oriented, hardworking and tenacious.

Neuroticism; includes traits such as being worried, depressive, angry, bored, sentimental, upset and insecure.

Openness to Experience; refers to solid imagination, being cultured and curious to learn, original, open-minded, clever and having artistic sensibility.

The study adopted 38-article "Extreme Sports Consumption Scale" as another data collecting tool, which was developed by Şimşek in 2010. The scale was a five-point Liker type consisting of answers ranging from $1=$ Strongly Disagree to $5=$ Strongly Agree. On the scale, scores were acquired from dimensions such as values, socialisation, seeking for excitement and adventure, letting of stress, physical fitness, perceived value, perceived satisfaction and behavioural intentions.

Values; refer to entertainment, freedom and self-esteem,

Socialisation; refers to an individual's consideration of a sport activity as an opportunity to interact with others (family, friends and spectators)

Excitement and adventure seeking; mostly refers to autonomous needs of an individual (getting pleasure, taking risks etc.)

Letting off stress; refers to an individual's departure from his or her problems and reaching to a better mood.

Physical fitness; refers to wish for living and looking physically well

Perceived value; refers to athletes' integrative assessment of benefits of goods and services concerning their sports branches.

Perceived satisfaction; is the satisfaction of athletes about the equipment that they use during completions and training.

Behavioural intentions; as a consequence of athletes' satisfaction with the equipment they use, refer to possible recommendations to others and further expenditures on these products.

\section{Findings}

Table 1: Distribution of Participants According to Gender

\begin{tabular}{|l|l|l|}
\hline Gender & $\mathbf{N}$ & $\mathbf{0}$ \\
\hline Female & 86 & 37.9 \\
\hline Male & 141 & 62.1 \\
\hline Total & $\mathbf{2 2 7}$ & $\mathbf{1 0 0}$ \\
\hline
\end{tabular}


Ceylan, M., Altıparmak, E., \& Akçakoyun, F. (2016). The analysis of the relationship between personality traits of extreme athletes and sports consumption motives. International Journal of Human Sciences, 13(1), 1745-1754. doi:10.14687/ijhs.v13i1.3416

Distribution of participants according to gender demonstrates that $37.9 \%$ (86) of the participants were women, $62.1 \%$ (141) were men.

Table 2: Distribution of Participants According to Sports Branches

\begin{tabular}{|l|l|l|l|l|l|l|}
\hline \multirow{2}{*}{ Branches } & \multicolumn{3}{|c|}{ N } & \multicolumn{3}{c|}{ \% } \\
\cline { 2 - 7 } & Female & Male & Total & Female & Male & Total \\
\hline Kiteboarding & 26 & 29 & 55 & 11.4 & 12.8 & 24.2 \\
\hline Windsurfing & 10 & 16 & 26 & 4.4 & 7.1 & 11.5 \\
\hline Paragliding & 2 & 15 & 17 & 0.8 & 6.7 & 7.5 \\
\hline Free-diving & 9 & 10 & 19 & 3.9 & 4.5 & 8.4 \\
\hline Snowboarding & 13 & 22 & 35 & 5.7 & 9.7 & 15.4 \\
\hline Snowkiting & 13 & 15 & 28 & 5.7 & 6.6 & 12.3 \\
\hline Rock Climbing & 3 & 11 & 14 & 1.3 & 4.9 & 6.2 \\
\hline Rafting & 3 & 8 & 11 & 1.3 & 3.5 & 4.8 \\
\hline Kayak & 2 & 8 & 10 & 0.8 & 3.6 & 4.4 \\
\hline Mountain Biking & 5 & 7 & 12 & 2.2 & 3.1 & 5.3 \\
\hline Total & 86 & 141 & 227 & 37.5 & 62.5 & 100 \\
\hline Grand Total & \multicolumn{2}{|l|}{} & $\mathbf{2 2 7}$ & & $\mathbf{1 0 0}$ & \\
\hline
\end{tabular}

Distribution of Participants According to sports branches are the following: Kiteboarding 24.2\% (55), Windsurfing $11.5 \%$ (26), Paragliding 7,5 (17), Free-diving $8.4 \%$ (19), Snowboarding $15.4 \%$ (35), Snowkiting12. $3 \%$ (28), Rock Climbing 6.2\% (14), Rafting $4.8 \%$ (11), Kayak 4.4\% (10) and Mountain Biking $5.3 \%$ (12).

Table 3: Distribution of Participants According to Marital Status

\begin{tabular}{|l|l|l|l|l|l|}
\hline \multirow{2}{*}{ Marital Status } & \multicolumn{2}{|c|}{ N } & \multicolumn{3}{c|}{ \% } \\
\cline { 2 - 6 } & Female & Male & Female & Male & Total \\
\hline Married & 21 & 43 & 9.2 & 19 & 28.2 \\
\hline Single & 53 & 65 & 23.3 & 28.7 & 52 \\
\hline Divorced & 12 & 33 & 5.2 & 14.6 & 19.8 \\
\hline Total & 86 & 141 & 37.7 & 62.3 & 100 \\
\hline Grand Total & \multicolumn{2}{|c|}{227} & \multicolumn{4}{c|}{$\mathbf{1 0 0}$} \\
\hline
\end{tabular}

According to their marital status, $28.2 \%$ (64) of participants were married, $52.0 \%$ (118) were single and $19.8 \%$ (45) were divorced.

Table 4: Distribution of Participants According to Education Level

\begin{tabular}{|l|l|l|l|l|l|}
\hline \multirow{2}{*}{ Education level } & \multicolumn{2}{|c|}{ N } & \multicolumn{3}{c|}{ \% } \\
\cline { 2 - 7 } & Female & Male & Female & Male & Total \\
\hline Literate & 1 & 0 & 0.4 & 0 & 0.4 \\
\hline Elementary School & 2 & 5 & 0.8 & 2.3 & 3.1 \\
\hline High School & 39 & 26 & 17.1 & 11.5 & 28.6 \\
\hline College/Bachelor's Degree & 28 & 90 & 12.3 & 39.7 & 52.0 \\
\hline Post-graduate (Masters and PhD.) & 16 & 20 & 7.0 & 8.9 & 15.9 \\
\hline Total & $\mathbf{8 6}$ & $\mathbf{1 4 1}$ & $\mathbf{3 7 . 6}$ & $\mathbf{6 2 . 4}$ & $\mathbf{1 0 0}$ \\
\hline Grand Total & \multicolumn{2}{|c|}{$\mathbf{2 2 7}$} & \multicolumn{4}{|c|}{$\mathbf{1 0 0}$} \\
\hline
\end{tabular}


Ceylan, M., Altıparmak, E., \& Akçakoyun, F. (2016). The analysis of the relationship between personality traits of extreme athletes and sports consumption motives. International Journal of Human Sciences, 13(1), 1745-1754. doi:10.14687/ijhs.v13i1.3416

The analysis of distribution of participants according to their education levels indicate that $.4 \%$ (1) of the participants were literate; 3.1\% (7) were elementary school graduates, $28.6 \%(65)$ were high school graduates, $52.0 \%$ (118) were college or university graduates and $15.9 \%$ (36) of the participants had post-graduate (masters and doctorate) degrees.

Table 5: The Comparison of FFPI Sub-dimensions According to Gender

\begin{tabular}{|l|l|l|l|l|l|l|}
\hline Dimensions of Personality & Gender & $\mathbf{N}$ & Mean & $\begin{array}{l}\text { Std. } \\
\text { Deviation }\end{array}$ & T & P \\
\hline \multirow{2}{*}{ Extraversion } & Female & 86 & 2,14 &, 35 & $-2,082$ &, $039 *$ \\
& Male & 141 & 2,24 &, 31 & & \\
\hline \multirow{2}{*}{ Docility } & Female & 86 & 2,12 &, 40 &,- 088 &, 930 \\
& Male & 141 & 2,12 &, 35 & & \\
\hline \multirow{2}{*}{ Self-Control } & Female & 86 & 2,40 &, 38 & $-1,242$ &, 216 \\
& Male & 141 & 2,45 &, 29 & & \\
\hline \multirow{2}{*}{ Neuroticism } & Female & 86 & 3,57 &, 51 &, 856 &, 393 \\
& Male & 141 & 3,51 &, 49 & & \\
\hline \multirow{2}{*}{ Openness to Experience } & Female & 86 & 2,06 &, 36 & $-1,638$ &, 103 \\
& Male & 141 & 2,15 &, 41 & & \\
\hline
\end{tabular}

$$
{ }^{*} \mathrm{p}<.05
$$

On table 5, in order to analyse differences between FFPI's sub-dimension scores in male and female sample groups, independent samples t- test was applied and a significant difference was observed concerning extraversion. The results showed that extraversion was much more in male sample group in comparison to female sample group.

Table 6: The Comparison of ESCM Sub-dimensions According to Gender

\begin{tabular}{|l|l|l|l|l|l|l|}
\hline Groups & Gender & $\mathbf{N}$ & Mean & Std.Deviation & $\mathbf{T}$ & $\mathbf{P}$ \\
\hline \multirow{2}{*}{ Values } & Female & 86 & 53,00 & 4,58 &,- 545 &, 586 \\
& Male & 141 & 53,31 & 3,92 & & \\
\hline \multirow{2}{*}{ Socialisation } & Female & 86 & 30,32 & 3,82 & $-1,406$ &, 161 \\
& Male & 141 & 30,98 & 3,17 & & \\
\hline Excitement- & Female & 86 & 29,45 & 3,85 &,- 217 &, 828 \\
Adventure & Male & 141 & 29,56 & 3,81 & & \\
\hline \multirow{2}{*}{ Letting off stress } & Female & 86 & 18,43 & 1,78 & 1,228 &, 221 \\
& Male & 141 & 18,13 & 1,74 & & \\
\hline \multirow{2}{*}{ Physical Fitness } & Female & 86 & 17,69 & 1,95 &,- 469 &, 640 \\
& Male & 141 & 17,81 & 1,76 & & \\
\hline \multirow{2}{*}{ Perceived Value } & Female & 86 & 13,60 & 1,20 &, 140 &, 889 \\
& Male & 141 & 13,58 & 1,20 & & \\
\hline \multirow{2}{*}{$\begin{array}{l}\text { Perceived } \\
\text { Satisfaction }\end{array}$} & Female & 86 & 17,46 & 1,21 & $-1,100$ &, 272 \\
\hline \multirow{2}{*}{ Behavioural } & Male & 141 & 17,67 & 1,48 & & \\
Intentions & Female & 86 & 24,09 & 2,17 &, 956 &, 340 \\
& Male & 141 & 23,80 & 2,26 & & \\
\hline
\end{tabular}

$*_{\mathrm{p}}<.05$ 
Ceylan, M., Altıparmak, E., \& Akçakoyun, F. (2016). The analysis of the relationship between personality traits of extreme athletes and sports consumption motives. International Journal of Human Sciences, 13(1), 1745-1754. doi:10.14687/ijhs.v13i1.3416

On table 6, t test was employed in order to analyse differences of ESCM sub-dimensions scores between male and female sample groups. The results indicated that there was no significant difference between male and female athletes in terms of extreme sports consumption motives.

\section{Table 7: The Relationship Between ESCM Sub-Dimensions and FFPI of Male Athletes}

\begin{tabular}{|c|c|c|c|c|c|c|c|c|c|c|c|c|c|}
\hline ----- & 1. & 2. & 3. & 4. & 5. & 6. & 7. & 8. & 9. & 10. & 11. & 12. & 13. \\
\hline 1.Extraversion & --- & & & & & & & & & & & & \\
\hline 2.Docility & $\begin{array}{c}, 181^{*} \\
, 031\end{array}$ & --- & & & & & & & & & & & \\
\hline 3.Self-control & $\begin{array}{c}, 270^{* *} \\
, 001 \\
\end{array}$ & $\begin{array}{c}247 * * \\
, 003 \\
\end{array}$ & --- & & & & & & & & & & \\
\hline 4.Neuroticism & $\begin{array}{l}-, 164 \\
, 052\end{array}$ & $\begin{array}{c}-, 399 * * \\
, 000\end{array}$ & $\begin{array}{c}-, 441^{* *} \\
, 000\end{array}$ & --- & & & & & & & & & \\
\hline $\begin{array}{l}\text { 5.Openness to } \\
\text { Experience }\end{array}$ & $\begin{array}{c}, 237^{* *} \\
, 005 \\
\end{array}$ & $\begin{array}{c}301 * * \\
, 000 \\
\end{array}$ & $\begin{array}{l}, 134 \\
, 113 \\
\end{array}$ & $\begin{array}{c}-, 184^{*} \\
, 029 \\
\end{array}$ & --- & & & & & & & & \\
\hline 6.Values & $\begin{array}{l}, 041 \\
, 626\end{array}$ & $\begin{array}{l}-, 059 \\
, 487\end{array}$ & $\begin{array}{l}, 016 \\
, 846\end{array}$ & $\begin{array}{l}, 063 \\
, 455\end{array}$ & $\begin{array}{l}, 026 \\
, 761\end{array}$ & --- & & & & & & & \\
\hline 7.Socialisation & $\begin{array}{l}, 056 \\
, 508 \\
\end{array}$ & $\begin{array}{l}-, 086 \\
, 312\end{array}$ & $\begin{array}{l}, 050 \\
, 555\end{array}$ & $\begin{array}{l}, 080 \\
, 348\end{array}$ & $\begin{array}{l}, 026 \\
, 761\end{array}$ & $\begin{array}{l}, 158 \\
, 061\end{array}$ & --- & & & & & & \\
\hline $\begin{array}{l}\text { 8.Excitement- } \\
\text { Adventure }\end{array}$ & $\begin{array}{l}-, 052 \\
, 540\end{array}$ & $\begin{array}{l}-, 032 \\
, 705\end{array}$ & $\begin{array}{l}119 \\
, 161\end{array}$ & $\begin{array}{l}-, 030 \\
, 723\end{array}$ & $\begin{array}{l}-, 022 \\
, 798\end{array}$ & $\begin{array}{c}, 231 * * \\
, 006\end{array}$ & $\begin{array}{l}146 \\
, 085\end{array}$ & --- & & & & & \\
\hline $\begin{array}{l}\text { 9.Letting off } \\
\text { stress }\end{array}$ & $\begin{array}{c}166^{*} \\
, 049\end{array}$ & $\begin{array}{l}, 019 \\
, 827\end{array}$ & $\begin{array}{l}-, 024 \\
, 780\end{array}$ & $\begin{array}{l}, 081 \\
, 342\end{array}$ & $\begin{array}{l}, 043 \\
, 612\end{array}$ & $\begin{array}{l}, 151 \\
, 075\end{array}$ & $\begin{array}{l}148 \\
, 080\end{array}$ & $\begin{array}{l}, 077 \\
, 364\end{array}$ & --- & & & & \\
\hline $\begin{array}{l}\text { 10.Physical } \\
\text { Fitness }\end{array}$ & $\begin{array}{l}123 \\
, 148\end{array}$ & $\begin{array}{l}, 021 \\
, 808\end{array}$ & $\begin{array}{l}-, 045 \\
, 598\end{array}$ & $\begin{array}{l}-, 052 \\
, 542\end{array}$ & $\begin{array}{l}, 077 \\
, 366\end{array}$ & $\begin{array}{l}, 053 \\
, 535\end{array}$ & $\begin{array}{l}117 \\
, 168\end{array}$ & $\begin{array}{l}, 079 \\
, 351\end{array}$ & $\begin{array}{c}, 421 * * \\
, 000\end{array}$ & --- & & & \\
\hline $\begin{array}{l}\text { 11.Perceived } \\
\text { Value }\end{array}$ & $\begin{array}{l}-, 005 \\
, 949\end{array}$ & $\begin{array}{l}-, 065 \\
, 445\end{array}$ & $\begin{array}{l}-, 163 \\
-054\end{array}$ & $\begin{array}{l}, 139 \\
, 101\end{array}$ & $\begin{array}{l}-, 112 \\
, 185\end{array}$ & $\begin{array}{l}-, 081 \\
, 341\end{array}$ & $\begin{array}{l}, 123 \\
, 145\end{array}$ & $\begin{array}{l}-, 035 \\
, 681\end{array}$ & $\begin{array}{l}, 034 \\
, 691\end{array}$ & $\begin{array}{l}, 114 \\
, 178\end{array}$ & --- & & \\
\hline $\begin{array}{l}\text { 12.Perceived } \\
\text { Satisfaction }\end{array}$ & $\begin{array}{l}031 \\
, 176\end{array}$ & $\begin{array}{l}-, 013 \\
, 877\end{array}$ & $\begin{array}{l}068 \\
, 426\end{array}$ & $\begin{array}{l}, 043 \\
, 614\end{array}$ & $\begin{array}{c}-, 092 \\
, 279\end{array}$ & $\begin{array}{l}099 \\
, 244\end{array}$ & $\begin{array}{l}, 025 \\
, 770\end{array}$ & $\begin{array}{l}-, 076 \\
, 373\end{array}$ & $\begin{array}{l}, 045 \\
, 597\end{array}$ & $\begin{array}{l}-, 018 \\
, 835\end{array}$ & $\begin{array}{l}, 005 \\
, 953\end{array}$ & -.-- & --- \\
\hline
\end{tabular}

The analysis of table 7 indicates that for male participant, there are positive correlations between ESCM letting off stress sub-dimension and extraversion as a personal trait $(r=, 166$, $\mathrm{p}<.05)$ and ESCM behaviouralintentions sub-dimension and extraversion as a personal trait $(\mathrm{r}$ $=, 167, \mathrm{p}<0.5)$. 
Ceylan, M., Altıparmak, E., \& Akçakoyun, F. (2016). The analysis of the relationship between personality traits of extreme athletes and sports consumption motives. International Journal of Human Sciences, 13(1), 1745-1754. doi:10.14687/ijhs.v13i1.3416

Table 8: The Relationship Between ESCM Sub-Dimensions and FFPI of Female Athletes

\begin{tabular}{|c|c|c|c|c|c|c|c|c|c|c|c|c|c|}
\hline ------ & 1. & 2. & 3. & 4. & 5. & 6. & 7. & 8. & 9. & 10. & 11. & 12. & 13. \\
\hline 1.Extraversion & --- & & & & & & & & & & & & \\
\hline 2.Docility & $\begin{array}{l}, 109 \\
, 320 \\
\end{array}$ & -- & & & & & & & & & & & \\
\hline 3.Self-control & $\begin{array}{c}, 245^{*} \\
, 023\end{array}$ & $\begin{array}{c}, 329 * * \\
, 002\end{array}$ & --- & & & & & & & & & & \\
\hline 4.Neuroticism & $\begin{array}{c}-, 198 \\
, 067\end{array}$ & $\begin{array}{c}-, 213^{*} \\
, 049\end{array}$ & $\begin{array}{c}-, 501 * * \\
, 000\end{array}$ & --- & & & & & & & & & \\
\hline $\begin{array}{l}\text { 5. Openness to } \\
\text { Experience }\end{array}$ & $\begin{array}{c}, 379 * * \\
, 000 \\
\end{array}$ & $\begin{array}{l}, 174 \\
, 109 \\
\end{array}$ & $\begin{array}{c}, 255^{*} \\
, 018 \\
\end{array}$ & $\begin{array}{c}-, 276^{*} \\
, 010 \\
\end{array}$ & --- & & & & & & & & \\
\hline 6.Values & $\begin{array}{l}, 039 \\
, 721 \\
\end{array}$ & $\begin{array}{c}-, 328 * * \\
, 002 \\
\end{array}$ & $\begin{array}{l}-, 174 \\
109 \\
\end{array}$ & $\begin{array}{l}, 023 \\
, 833 \\
\end{array}$ & $\begin{array}{l}-, 024 \\
, 827 \\
\end{array}$ & --- & & & & & & & \\
\hline 7.Socialisation & $\begin{array}{l}, 127 \\
, 244 \\
\end{array}$ & $\begin{array}{c}-, 397 * * \\
, 000 \\
\end{array}$ & $\begin{array}{l}-, 136 \\
, 213 \\
\end{array}$ & $\begin{array}{l}, 046 \\
, 676 \\
\end{array}$ & $\begin{array}{l}, 021 \\
, 847 \\
\end{array}$ & $\begin{array}{c}, 435 * * \\
, 000\end{array}$ & -- & & & & & & \\
\hline $\begin{array}{l}\text { 8.Excitement- } \\
\text { Adventure }\end{array}$ & $\begin{array}{l}, 069 \\
, 527 \\
\end{array}$ & $\begin{array}{c}-, 214 * \\
, 048\end{array}$ & $\begin{array}{l}-, 011 \\
, 920 \\
\end{array}$ & $\begin{array}{l}-, 105 \\
, 337\end{array}$ & $\begin{array}{l}\text {,064 } \\
, 559\end{array}$ & $\begin{array}{c}, 351^{* * *} \\
, 001 \\
\end{array}$ & $\begin{array}{l}, 218^{*} \\
, 044\end{array}$ & --- & & & & & \\
\hline $\begin{array}{l}\text { 9.Letting off } \\
\text { stress }\end{array}$ & $\begin{array}{c}-, 079 \\
, 472 \\
\end{array}$ & $\begin{array}{l}-, 042 \\
, 702 \\
\end{array}$ & $\begin{array}{c}-, 270^{*} \\
, 012 \\
\end{array}$ & $\begin{array}{l}, 100 \\
, 362\end{array}$ & $\begin{array}{c}-, 003 \\
, 981\end{array}$ & $\begin{array}{l}, 208 \\
, 054\end{array}$ & $\begin{array}{l}, 184 \\
, 089\end{array}$ & $\begin{array}{r}-, 037 \\
, 734 \\
\end{array}$ & -- & & & & \\
\hline $\begin{array}{l}\text { 10.Physical } \\
\text { Fitness }\end{array}$ & $\begin{array}{c}-, 251^{*} \\
, 020 \\
\end{array}$ & $\begin{array}{c}-, 182 \\
, 093\end{array}$ & $\begin{array}{l}-, 203 \\
, 061 \\
\end{array}$ & $\begin{array}{l}, 142 \\
, 192 \\
\end{array}$ & $\begin{array}{r}-, 025 \\
, 822 \\
\end{array}$ & $\begin{array}{c}, 323 * * \\
, 002\end{array}$ & $\begin{array}{c}, 251^{*} \\
, 020\end{array}$ & $\begin{array}{l}, 250^{*} \\
, 021\end{array}$ & $\begin{array}{c}, 365^{* * *} \\
, 001\end{array}$ & -- & & & \\
\hline $\begin{array}{l}\text { 11.Perceived } \\
\text { Value }\end{array}$ & $\begin{array}{c}-, 139 \\
, 203 \\
\end{array}$ & $\begin{array}{l}, 190 \\
, 081 \\
\end{array}$ & $\begin{array}{l}-, 074 \\
, 497 \\
\end{array}$ & $\begin{array}{l}, 128 \\
, 240\end{array}$ & $\begin{array}{l}-, 100 \\
, 359 \\
\end{array}$ & $\begin{array}{l}-, 062 \\
, 571 \\
\end{array}$ & $\begin{array}{l}, 021 \\
, 850 \\
\end{array}$ & $\begin{array}{l}-, 160 \\
, 333 \\
\end{array}$ & $\begin{array}{l}, 102 \\
, 349 \\
\end{array}$ & $\begin{array}{l}, 109 \\
, 318 \\
\end{array}$ & --- & & \\
\hline $\begin{array}{l}\text { 12.Perceived } \\
\text { Satisfaction }\end{array}$ & $\begin{array}{l}, 197 \\
, 068\end{array}$ & $\begin{array}{l}-, 055 \\
, 612\end{array}$ & $\begin{array}{l}, 078 \\
, 476\end{array}$ & $\begin{array}{l}-, 067 \\
, 542 \\
\end{array}$ & $\begin{array}{l}\text {,092 } \\
, 397\end{array}$ & $\begin{array}{l}, 089 \\
, 416\end{array}$ & $\begin{array}{l}, 030 \\
, 781\end{array}$ & $\begin{array}{l}-, 053 \\
, 627\end{array}$ & $\begin{array}{l}-, 007 \\
, 952\end{array}$ & $\begin{array}{l}-, 084 \\
, 443\end{array}$ & $\begin{array}{l}, 152 \\
, 163\end{array}$ & --- & --- \\
\hline $\begin{array}{l}\text { 13. Behavioral } \\
\text { Intentions }\end{array}$ & $\begin{array}{l}, 075 \\
, 494\end{array}$ & $\begin{array}{l}, 059 \\
, 591 \\
\end{array}$ & $\begin{array}{l}, 010 \\
, 930\end{array}$ & $\begin{array}{l}-, 091 \\
, 406\end{array}$ & $\begin{array}{l}-, 069 \\
-530\end{array}$ & $\begin{array}{l}-, 086 \\
, 430\end{array}$ & $\begin{array}{c}-, 032 \\
, 770\end{array}$ & $\begin{array}{l}, 041 \\
, 706\end{array}$ & $\begin{array}{c}-, 062 \\
, 571\end{array}$ & $\begin{array}{l}\text {,018 } \\
, 871\end{array}$ & $\begin{array}{l}, 104 \\
, 339\end{array}$ & $\begin{array}{l}, 113 \\
, 302\end{array}$ & - \\
\hline
\end{tabular}

As Table 8 indicates, for female participants negative correlations were found between ESCM physical fitness sub-dimension and extraversion as a personal trait $(r=-, 251, \mathrm{p}<.05)$, ESCM values sub-dimension and docility as a personal trait $(\mathrm{r}=-, 328, \mathrm{p}<, .01)$, ESCM socialisation subdimension and docility as a personal trait $(\mathrm{r}=-, 397, \mathrm{p}<.01)$, ESCM excitement-adventure subdimension and docility as a personal trait $(\mathrm{r}=-, 214, \mathrm{p}<.05)$ and between ESCM letting off stress sub-dimension and self-control as a personal trait $(r=-, 270, \mathrm{p}<.05)$.

\section{Discussion}

The research aimed to analyse the impacts of personal traits of extreme athletes on their sports consumption motives. A total number of 227 athletes (141 males, $62.1 \%$ and 86 females, $37.9 \%$ ) participated in this research (Table 1). Dökmen (2006) states that in Turkish society, boys are directed to more rough motor skills, enterprisingness and recklessness whereas girls were directed to more gentle and delicate motor skills. Our research consists of more male athletes; this fact points out that female athletes are mostly encouraged for more traditional sports as male athletes are more common and bigger in number concerning extreme sports.

Athletes, who are active competitors or who train for a competition, deal with the following branches: 26 windsurfing (10 females, 16 males), 54 kitesurfing (26 females 29 males), 35 snowboarding(13 females, 22 males), 28 snow kiting (13 females, 15 males), 10 Kayak ( 2 females, 8 males), 14 Rock Climbing ( 3 females, 11 males), 19 Free-diving ( 9 females, 10 males), 11 Rafting (3 females, 8 males), 12 Mountain Biking (5 Female, 7 Male)and17 Paragliding ( 2 females, 15 males)(Table 2). Particularly the branch of kiteboarding, which is considered as the most rapid developing sport in the last decade, due to its easiness for candidates to become competitors (Ceylan, 2010), includes more athletes than others; therefore it is thought that most participants in this research deal with this sport.

According to their marital status, $28.2 \%$ (64) of participants were married, $52.0 \%$ (118) were single and $19.8 \%$ (45) were divorced (Table 3). Education levels of the participants indicate 
Ceylan, M., Altıparmak, E., \& Akçakoyun, F. (2016). The analysis of the relationship between personality traits of extreme athletes and sports consumption motives. International Journal of Human Sciences, 13(1), 1745-1754. doi:10.14687/ijhs.v13i1.3416

that extreme athletes have high levels of education; $52 \%$ of them have college and bachelor's degree (Table 4). Sönmez (2006) in his study on windsurfers demonstrated that education levels of windsurfers are high. This result is compatible with our research findings. Extreme sports require high technique, theoretical knowledge and skills; therefore it is considered that sports consumers with higher education levels are interested in these branches.

When the difference between FFPI sub-dimension scores in male and female sample groups was analysed, a significant difference was observed with regard to extraversion (Table 5). According to the results, males are more extraverted than females. Extraversion consists of traits such as being energetic, talkative, sympathetic, excited, enthusiastic and social. Rauter (2011) in his research acknowledges that male athletes receive more solid reinforces than female do and this situation leads male athletes to be more extraverted and self-confident in terms of sportive talents. ESCM sub-dimension scores for male and female sample groups were also examined. Accordingly there was no significant difference between male and female athletes in terms of extreme sports consumption motives (Table 6).

When the relationship between ESCM and FFPI was analysed according to the gender factor, for female participants negative correlations were found between physical fitness and extraversion; values, socialisation, seeking for excitement-adventure and docility and letting of stress and self-control (Table 8). Extravert individuals are generally social, assertive, active and talkative, ambitious and friendly. They are sympathetic and they seek for adventure. Basic motivation factors of extravert individuals are being superior and achievement oriented. Introvert individuals, who are on the other extreme of the extraversion sub-dimension, are tend to be shy, timid and quiet (Werch,1997). Some scholar mention that the primary factor motivating sports participation is physical fitness, which is an important motivating factor alongside with self-esteem, talent development and friendship. In this perspective, it is considered that physical fitness of an individual influences his or her extraversion.

Letting off stress is regarded as one of the important factors for participation and consumption of sports. There is an extensive research on this issue. Most of these studies indicate that increase in exercise leads to a decrease stress level. Mete (2006) refers self-control dimension of personality as being planned and patient towards target-oriented behaviours, motivation, success and concepts that are associated with this factor. Individuals with these traits are strong-willed and determined. Being conscious includes being success-oriented, ordered, effective, organisational, planned, responsible, perfectionist and hardworking. Some researchers consider this factors as a wish to be successful; some consider these people as self-organised and with the habit of being careful and talent of self-discipline. This dimension shows the level of control and discipline. This personal trait refers to responsible, dependent, careful, disciplined, achievement-oriented and determined personality. Individuals with these traits possibly have strong chances to be successful in any task in both autonomous and hierarchical structure. Burger (2006) defines individuals with low responsibility as careless, easily distracted, unreliable persons, who are easy in reaching their targets. Instinctive individuals, who are located on the negative extreme of the self-control dimension, are more disorderly, careless; they keep themselves out of control. In our research, as participants were competitor individuals, female competitors do sports not only let off steam but also to achieve success.

Again, for female athletes a negative correlation between ESCM's sub dimensions of socialisation, seeking for excitement-adventure and values and FFPI's sub dimension of docility. Traits like value perception, the need for socialisation, seeking for excitement and adventure refer to more autonomous needs on individuals, docility refers to trustable, well-mannered, prone to cooperation, forgiving, light-hearted, tolerant; it underlines the social side of an individual, who have emotional ties with others (Allman et. al., 2009). Docile individuals are known as being sincere, sympathetic, cooperative, trustable; they pay more attention to cooperation rather than competition and they tend to help other individuals. The individuals, whose docility levels are low, 
Ceylan, M., Altıparmak, E., \& Akçakoyun, F. (2016). The analysis of the relationship between personality traits of extreme athletes and sports consumption motives. International Journal of Human Sciences, 13(1), 1745-1754. doi:10.14687/ijhs.v13i1.3416

are usually stubborn, competitive, incompatible; it is hard to settle with them. Due to the fact that extreme sports are individual sports, individuals are more "self" oriented and it can be said that they do extreme sports to satisfy their individual desires and wishes rather than to socialise.

When the relationship between ESCM and FFPI was analysed according to the gender factor, for male participants positive correlations were found between sub-dimensions of letting off steam and behavioural intensions and extraversion as a personal trait (Table 9). Heinström (2003) defines extravert individuals as social persons who like to participate several groups and parties; they have many friends, they do not like to stay alone, study or read alone. Extraverts prefer excitement and euphoria; they do not hesitate to engage in dangerous situations. They can decide and act rapidly. They like to crack a joke and to talk. They easily make communication and friends. They are easy going, optimistic people; they fancy to laugh and to make others laugh. In comparison to introverts, extraverts have more positive effects, more expectations for positive events, stronger reaction and energy (Kajtna et. al., 2004).

Behavioural intentions, as a consequence of athletes' satisfaction with the equipment they use, refer to possible recommendations to others and further expenditures on these products. The trust to equipment's reduces material-oriented risk to minimum as well as the stress factor to the lowest level. Therefore utility that is obtained from the extreme sports reaches the maximum level. In addition it is considered that an individual's trust to his or her equipment increases his or her self-confidence. This increase in confidence is thought to lead more interaction and share with others.

\section{Conclusion}

The results of the research indicate that sports consumption movies coincide with personal traits to some extent. The research included athletes, who did not participate extreme sports as recreational activities but as competitions. Being a competitor in an extreme sport branch after a while causes different perception of the concept "extreme" by the athlete. This changes his or her perception of risk and danger. As individuals spend more time in doing extreme sports, extraordinary things gradually turn into ordinary things. For this reason, it is suggested that future studies would analyse the time consumed for extreme sports as a variable.

Moreover, participants answered questions in questionnaires in their own sportive environment. As it is known, most extreme sports take place as long as there are proper weather conditions. Some extreme athletes, who did not want to miss proper weather conditions were thought to answer in hurry; some of them were considered that they did not totally understand questions due to fatigue. Therefore, it is more convenient for further research on this issue to collect data after training and seasons.

\section{References}

1. Allman, T.L., Mittelstaedt,R.D., Martin, B., Goldenberg, M., (2009). "Exploring the Motivations of BASE Jumpers: Extreme Sport Enthusiasts", Journal of Sport \& Tourism Vol. 14, No. 4, , pp. 229-247

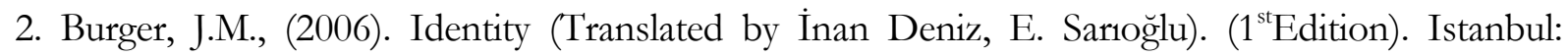
Kaktüs Publications

3. Ceylan M., Tekin A., Özdağ S., Ceylan Ö., (2010). "Uçurtma Ve Rürgar Sörfü Yapan Bireylerin Baž Kişilike Özelliklerinin Karşılaștırlması", Türkiye Kickboks Federasyonu Spor Bilimleri Dergisi Say1:2,

4. Cüceloglu, D., (2007). İnsan ve Davranışı: Psikolojinin Temel Kavramları, p.405, Istanbul 
Ceylan, M., Altıparmak, E., \& Akçakoyun, F. (2016). The analysis of the relationship between personality traits of extreme athletes and sports consumption motives. International Journal of Human Sciences, 13(1), 1745-1754. doi:10.14687/ijhs.v13i1.3416

5. Dökmen, Z., (2006). "Working, gender and gender roles and relation between housework and depression". Turkish Psychology J., 12(39): 39-56

6. Heinström, J., (2003) "Five Personality Dimensions and Their Influence on Information Behaviour", Department of Social and Political Sciences/Information Studies Abo Akademi University, $9(1): 165$

7. Huhra, R.L., (2007). "Religious Coping as A Moderator of The Five Factor Model of Personality Traits and Alcohol Abuse Severity At Six-Month Follow-Up in A Twelve Step Treatment Sample", The Graduate Faculty of The University of Akron, p.39

8. Kajtna, T., Tusak, M., Baric, R., Burnik, S., (2004). "Personality in high risk sports atbletes", Kinesiology, 36, 1:24-34

9. Kuru, E., (2003). “Farkh Statüdeki Beden Eğitimi Bölümü Öğrencilerinin Kişilik Özelliklern”, G.Ü. Gazi Eğitim Fakültesi Dergisi 23(1):175-191

10. Mete, C., (2006). "Illkögrretim Okullarnda Calşan Öğretmenlerin Kişilik Özellikleri İle İs Tatminleri Arasindaki İliskinin Incelenmesi” Yüksek Lisans Tezi, Yeditepe Üniversitesi, Sosyal Bilimler Enstitüsü, İstanbul

11. Palmer C., (2001). "Extreme sports a risky business for tourists". Media Release,11September 2000. Retrieved May 4

12. Somer, O., Korkmaz, M., Tatar, A., (2004). "Kuramdan Uygulamaya Beş Faktör Kişilike Modeli ve Beş Faktör Kişilik. Envanteri (5FKE) İzmir, Ege Üniversitesi Yayınlanı, s.1, 13-28, 47-49

13. Sönmez, U., (2006). "Rürgâr Sörfünün Yaylması Açısindan, Sörf Tüketicisinin Profilinin Belirlenmesi Ve Sörf Okullarmm Önemi” Yüksek Lisans Tezi, Manisa

14. Şimşek, K.Y., (2011). "Ekestrem Ve Geleneksel Spor Katılimcilar Arasinda Duygu Arama Farklhlklarmin Karşılaștırlması” Niğde Üniversitesi Beden Eğitimi Ve Spor Bilimleri Dergisi Cilt 5, Say1 2, 133-143

15. Şimşek, K.Y., (2010). "Türk Ekstrem Sporcular İçin Spor Tüketim Güdüsü Ölçeğinin Güvenirlike ve Geşerlike Calıs̆ması”, Celal Bayar Üniversitesi Beden Eğitimi ve Spor Bilimleri Dergis, 5(3):97106

16. Williams, L., Gill, D.L., (2000). "Cognitive Approaches to Motivation. Psychological Dynamics of Sport and Exercise”. Edt. Diane L. Gill. Human Kinetics, 2nd edition. Champaign, IL, 2000 